(including anxiety and depression) together with headache and/or eyestrain, and showed higher scores among women with higher educational level, white-collar jobs, and in the public sector. PC3 included the item on other disorders (comprising neurological, endocrine, autoimmune and voice-related) with some loading from digestive and hearing disorders, and showed higher scores in older and blue-collar workers. PC4 grouped respiratory with infectious diseases; it associated with working in the public sector and reporting work-related accident history. PC5 was composed by circulatory system conditions and associated with former smoking, higher BMI, and white-collar jobs.

Conclusion Work-related diseases aggregated in five distinct components, supporting the need for a shift from a disease-by-disease approach towards a worker-centered approach.

\section{O6A.2 BREAST CANCER RISK IN 40.000 DANISH WOMEN BY INDUSTRY}

Julie Elbæk Pedersen*, Johnni Hansen. The Danish Cancer Society Research Center, Copenhagen, Denmark

\subsection{6/OEM-2019-EPI.137}

Background Occupational exposures have been hypothesized as contributors to breast cancer. Nevertheless, studies exploring the risk of breast cancer by occupation or industry generally show somewhat inconsistent results. In addition to small study size and lack of lifetime work history, potential confounders, such as reproductive history, have often not been taking into account and mortality has often been used as outcome. We conducted a nationwide register based nested case-control study in order to explore associations between occupation and incidence of breast cancer, including reproductive history.

Methods A total of 38,347 employed women born in Denmark during the period 1946 to 1995 , and diagnosed with breast cancer 1970-2015 were identified in the nationwide Danish Cancer Registry. Five control subjects, work active and born in Denmark, and free of breast cancer at the date of diagnosis of the corresponding case, were chosen randomly from the Danish Civil Register. Using the unique personal identification number assigned to all residents in Denmark, we obtain lifetime employment history from the nationwide Pension Fund Register, which on an individual level has kept detailed information on all employments in Denmark since 1964. Lastly, information on reproductive factors, and socioeconomic status was retrieved from the Danish Civil Register. Odds ratios (ORs) with 95\% confidence intervals (CIs) were estimated by conditional logistic regression analysis.

Preliminary results: Risk elevations were e.g. seen in the military $(2.1 ; 1.49-2.90)$, printing $(1.6 ; 1.22-2.06)$, airline transportation $(2.4 ; 1.33-4.47)$, automobile service $(1.3 ; 95 \%$ CI 1.12-1.49), laundries (2.4; 1.48-3.79), hairdressing (1.9; $1.11-3.23)$ and paint factories (1.6; 95\% CI 1.22-2.06). Risk reductions were seen in farming $(0.4 ; 0.21-0.59)$, window cleaning $(0.6 ; 0.44-0.84)$ and building and carpentry $(0.8$; 0.70-0.98).

Conclusions The study suggests that breast cancer risk varies significantly by industry and that occupational exposures, including night-shift work, solvents and outdoor work, may play a possible role.

\section{A.3 IMPLICATIONS OF THE WORKPLACE GENDER COMPOSITION FOR DEPRESSION-RELATED SERVICE UTILIZATION: A RETROSPECTIVE COHORT STUDY OF U.S. ALUMINUM WORKERS}

${ }^{1}$ Holly Elser*, ${ }^{2}$ David Rehkopf, ${ }^{1}$ Patrick Bradshaw, ${ }^{3}$ Carmen Brick, ${ }^{3}$ Daniel Schneider, ${ }^{4}$ Ellen Eisen, ${ }^{5}$ Mark Cullen. ${ }^{1}$ UC Berkeley School of Public Health, Division of Epidemiology, Berkeley, USA; ${ }^{2}$ School of Medicine, Department of Medicine, Division of Primary Care and Population Health, Stanford University, Stanford, USA; ${ }^{3}$ UC Berkeley, Department of Sociology, Berkeley, USA; ${ }^{4}$ UC Berkeley School of Public Health, Division of Environmental Health Sciences, Berkeley, USA; ${ }^{5}$ Center for Population Health Sciences, Stanford University, Palo Alto, 94305

\subsection{6/OEM-2019-EPI.138}

Introduction Past research finds that both male and female workers in male-dominated workplaces experience decreased job satisfaction and increased work-related stress. Female workers in particular are more likely to experience gender discrimination, sexual harassment, and social isolation. Nevertheless, systematic evidence regarding the association between workplace gender composition and worker mental healthcare utilization remains limited.

Methods Using data from the American Manufacturing Cohort Study (AMC), we examined the association between workplace gender composition and risk of depression-related outpatient visits among hourly workers employed at one of 32 U.S. aluminum plants between 2003 - 2013.Using generalized additive models (GAM) with a logit link, we modeled risk of depression-related outpatient visits as a function of annual workplace gender composition (i.e. percent women) and covariates (baseline age, race, sex, plant, and calendar year). We captured potential nonlinear relationships using cubic smoothing splines. We used g-computation to summarize the counterfactual risk of depression-related outpatient visits under three hypothetical interventions wherein women comprised at least 10,20 , or 30 percent of the workforce at all plants for all years of followup. We compared these counterfactual risks to the risk under no intervention (the observed data).

Results Across all 32 U.S. plants, the final study sample included 5,279 female and 24,124 male hourly workers. We observed significant reductions in the number of depressionrelated outpatient visits when women comprised at least $20 \%$ $(\mathrm{RD}=-6.2,95 \% \mathrm{CI}-8.75,-3.65)$ and $30 \%(-14.6 ; 95 \% \mathrm{CI}-$ $19.0,-10.2)$ of the workforce. Findings were consistent within subgroups of male and female workers.

Conclusion The gender composition of industries, occupations, and workplaces is an important expression of social structure. We find that modest increases in the percent women employed in otherwise male-dominated work environments can lead to measurable reductions in the frequency of depression-related outpatient visits among male and female hourly workers.

\section{A.4 RISK OF POSTMENOPAUSAL BREAST CANCER AND OCCUPATIONAL EXPOSURE TO CHEMICALS IN SWEDISH WOMEN}

\footnotetext{
${ }^{1,2}$ Per Gustavsson*, 'Cecilia Videnros, 1,2Pernilla Wiebert, 'Nils Plato, ${ }^{3}$ Signe Borgquist, ${ }^{3,4}$ Jonas Manjer, 1,2Maria Albin, ${ }^{1} J$ Jenny Selander. 'Institute of Environmental Medicine, Karolinska Institutet, Stockholm, Sweden; ${ }^{2}$ Centre for Occupational and Environmental Medicine, Stockholm County Council, Stockholm, Sweden; ${ }^{3}$ Department of Clinical Sciences, Malmö, Lund University, Malmö, Sweden; ${ }^{4}$ Department of Surgery, Skåne University Hospital, Malmö, Sweden
}

10.1136/OEM-2019-EPI.139 